\title{
The "Boomerang" Malleus-Incus Complex in Congenital Aural Atresia
}

\author{
S. Mukherjee, B.W. Kesser, and P. Raghavan
}

\begin{abstract}
SUMMARY: "Boomerang" malleus-incus fusion deformity is identified on axial high-resolution CT in a subset of patients with congenital aural atresia, and it is associated with an absent incudostapedial joint and stapes capitulum and attachment of the incus to the tympanic segment of the facial nerve canal. Twelve patients with this deformity were identified on a retrospective review of imaging from a cohort of 673 patients with congenital aural atresia, with surgical confirmation in 9 of these patients. Eight of 9 patients underwent partial ossicular replacement prosthesis reconstruction with improvement in hearing outcome. We hypothesize that the boomerang anomaly represents a more severe ossicular anomaly than is normally seen in congenital aural atresia, arising from an arrest earlier in the embryonic development of the first and second branchial arch. This has potentially important implications for surgical planning because hearing outcomes with placement of prosthesis may not be as good as with conventional atresia surgery, in which reconstruction is performed with the patient's native ossicular chain.
\end{abstract}

ABBREVIATIONS: $\mathrm{CAA}=$ congenital aural atresia; $\mathrm{HRCT}=$ high-resolution $\mathrm{CT}$; $\mathrm{PORP}=$ partial ossicular replacement prosthesis

C ongenital aural atresia (CAA) is a rare disorder of the temporal bone with an estimated incidence of 1 in 10-20,000 live births. ${ }^{1}$ CAA refers to a spectrum of predominantly external and middle ear abnormalities resulting from incomplete or arrested development of the first and sometimes second branchial arches. The anomaly includes a failure of canalization of the external auditory canal with varying degrees of atresia, or underdevelopment, of the middle ear and ossicles. Depending on the timing of the arrest in development, the ear canal can be completely absent or mildly stenotic, with a wide range of ossicular malformations most commonly involving the malleus and incus. ${ }^{2}$

While patients with CAA have a wide variety of ossicular malformations and configurations seen on high-resolution CT (HRCT), we present a series of 12 patients with a characteristic, consistent "boomerang"-shaped fusion abnormality of the malleus and incus on axial sections through the epitympanum. In this anomaly, the malleus is hypoplastic, with an absent neck, manu-

Received January 23, 2014; accepted after revision May 3.

From the Departments of Radiology and Medical Imaging (S.M.) and OtolaryngologyHead and Neck Surgery (B.W.K.), University of Virginia Health System, Charlottesville, Virginia; and Department of Diagnostic Radiology and Nuclear Medicine (P.R.), University of Maryland School of Medicine, Baltimore, Maryland.

Drs Mukherjee and Kesser are co-first authors.

Please address correspondence to Sugoto Mukherjee, MD, Department of Radiology and Medical Imaging, Division of Neuroradiology, University of Virginia Health System, PO Box 800170, Charlottesville, VA 22908-0170; e-mail: Sm5qd@virginia.edu

http://dx.doi.org/10.3174/ajnr.A4022 brium, and umbo. The incus is the dominant ossicle and is fused to a rudimentary, hypoplastic malleus head to form the boomerang. This abnormality is accompanied by absence/hypoplasia of the stapes capitulum and ossicular discontinuity, with a fibrous attachment of the dysplastic incus to the tympanic segment of the facial nerve canal (Fig 1).

Recognition of this characteristic imaging finding can alert the radiologist and otologic surgeon to the presence of the associated findings. Because the incus is not attached to the stapes, this deformity will most often require a partial ossicular replacement prosthesis (PORP) reconstruction; hence, this finding should be communicated to the referring surgeon for optimal clinical decision-making, surgical planning, and patient counseling.

\section{MATERIALS AND METHODS \\ Patients}

This study was approved by the institutional review board of the University of Virginia, which waived consent for the study (IRBHSR 16763). Data were collected retrospectively from a chart and radiographic review, obviating individual patient consent. All data were de-identified in compliance with the institutional review board and Health Insurance Portability and Accountability Act regulations.

The cohort of 12 patients was collected through a retrospective search of electronic medical records of all patients diagnosed with CAA at our institution from 2001 to 2012, which was cross-referenced with the University of Virginia PACS imaging data base. 


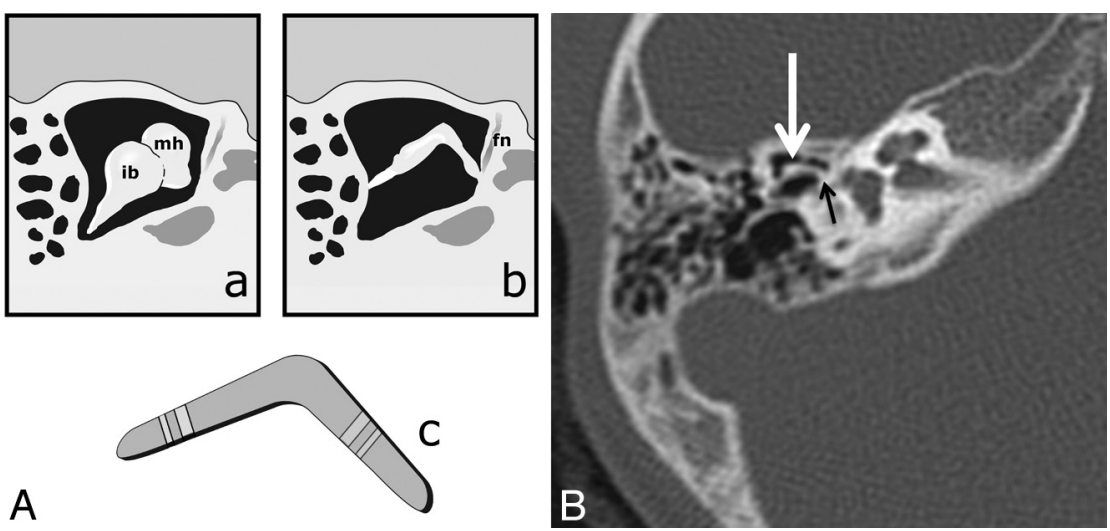

FIG 1. Schematic drawing (A) showing the normal appearance (a) of the malleus head ( $\mathrm{mh}$ ) and incus body (ib) and the abnormal fused dysplastic malleus-incus complex, appearing as a boomerang (b) as seen on axial CT images. Note the similarity to the appearance of a real boomerang (c). Axial HRCT scan (B) in patient 4 demonstrates the fused boomerang-shaped malleus head and incus body in the epitympanum (white arrow) and the attachment of the malleus-incus complex to the tympanic segment of the facial nerve canal (black arrow), rather than the stapes suprastructure. Also note the lateral attachment of the boomerang to the atretic plate. Findings were confirmed at surgery. fn indicates the location of tympanic segment of the facial nerve canal.

The search revealed 673 patients (770 ears) with CAA. Inclusion criteria were a diagnosis of CAA, an accessible HRCT on PACS (all outside films were loaded into the PACS system of the University of Virginia), and an identified boomerang malleus-incus complex either at surgery or, if the patient did not have surgery, through the author's (B.W.K.) CAA data base (UVA IRB 11484). Exclusion criteria included poor image quality secondary to motion artifacts and section width of $>1.25 \mathrm{~mm}$. All imaging studies were performed with the same protocol (see below), established by the surgeon to optimize evaluation for surgical candidacy. Because several of the studies were from multiple outside institutions, exposure parameters and radiation dosages were not collected. No patient had a named syndrome.

The neuroimaging was reviewed by all authors together (who, by the nature of the finding, were not blinded to the presence of this anatomic abnormality), and the findings were reached by consensus and tabulated. All authors (2 attending neuroradiologists and 1 otologic surgeon) had a minimum of 10 years' experience reviewing temporal bone images. All imaging studies were performed for assessing the patient's suitability for canaloplasty surgery. Demographic data, Jahrsdoerfer grade, and clinical, radiographic, and surgical findings (in 9 of 12 patients) were gathered. Three patients elected not to have surgery, so the radiographic anatomy could not be surgically confirmed.

\section{CT Technique}

All subjects in our institution had imaging performed on a 16section LightSpeed Pro multidetector scanner (GE Healthcare, Milwaukee, Wisconsin). The raw data were acquired helically with $0.625-\mathrm{mm}$ collimation at $300 \mathrm{mAs}$ and $120 \mathrm{kV}$ (peak), with coverage from the arcuate eminence through to the mastoid tip. The raw data from each ear were separated and reconstructed into 0.6- (section thickness) to $0.2-\mathrm{mm}$ (reconstruction interval) axial images in bone algorithms.

All outside HRCT studies were acquired helically on multidetector CT scanners by using $0.625-$ to $1.0-\mathrm{mm}$ section thickness and $0.3-\mathrm{mm}$ interval reconstructions in bone algorithms. Coronal and sagittal $(1.5 \times 1$ $\mathrm{mm}$ thickness) reconstructions were obtained as per the outside protocol.

\section{Image Analysis}

The HRCT images were interpreted in consensus by 3 experienced authors (S.M., P.R., and B.W.K.). The images were initially analyzed in the axial plane for the presence of the characteristic boomerangshaped fusion abnormality of the malleus-incus complex in the epitympanum and any other associated abnormalities in the rest of the middle ear. The findings were then confirmed on multiplanar reformats. The Jahrsdoerfer grading scale was used to determine surgical candidacy and served as a template for the radiologic review, ${ }^{1}$ with particular attention paid to the oval window, incudostapedial joint, stapes bone, and course of the facial nerve.

Imaging characteristics were correlated with ossicular and middle ear anatomy seen at the time of surgery for those patients undergoing surgical repair.

\section{Audiometric Outcomes}

Pure tone average (defined as the average of the air-conduction thresholds at 500,1000, 2000, and $4000 \mathrm{~Hz}$ ), speech reception thresholds (minimum intensity of sound [threshold] at which a patient is able to repeat $50 \%$ of a spondee word list), and air-bone gap (difference between the air-conduction pure tone average and the bone-conduction pure tone average) were collected and compared between the preoperative and postoperative values.

\section{RESULTS}

We identified 12 patients with CAA (10 males and 2 females) of 673 patients (770 ears) with the fused malleus-incus complex resembling a boomerang shape for a prevalence rate of 1.6 per 100 atretic ears. The ages of the patients ranged from 3 to 23 years. We obtained clinical and imaging data in all patients. Surgical confirmation was obtained in 9 of the 12 patients; 3 patients elected not to have surgery. All patients had grade III microtia, and all patients had complete atresia of the external auditory canal. Ten patients had unilateral CAA, with 2 patients having bilateral CAA. The boomerang deformity was unilateral in all patients. Demographic, clinical, and imaging findings are summarized in Table 1.

The characteristic fused boomerang malleus-incus complex was identified in all 12 patients on axial sections in the epitympanum (Fig 1). In this anomaly, the malleus is hypoplastic, with an absent neck, manubrium, and umbo. The incus is the dominant ossicle and is fused to a rudimentary, hypoplastic malleus head forming the boomerang. The boomerang complex also showed attachment laterally to the atretic tympanic plate in 9 patients and medially to the tympanic segment of the bony facial nerve canal in all patients (Figs 1 and 2). Coronal imaging shows mostly the incus with a hypoplastic malleus head and the absence of the incudostapedial joint at its expected location adjacent to the oval 
Table 1: Imaging and clinical findings in patients with boomerang malleus-incus complex in patients with CAA

\begin{tabular}{|c|c|c|c|c|c|c|c|c|c|c|}
\hline $\mathrm{Pt}$ & $\begin{array}{l}\text { Age } \\
\text { (yr) }\end{array}$ & Sex & Side & $\begin{array}{l}\text { Boomerang } \\
\text { Malleus-Incus } \\
\text { Complex }\end{array}$ & $\begin{array}{l}\text { Attachment to } \\
\text { Tympanic Segment } \\
\text { of Facial Canal }\end{array}$ & $\begin{array}{l}\text { Absent/Dysmorphic } \\
\text { Stapes Capitulum }\end{array}$ & $\begin{array}{l}\text { Oval Window } \\
\text { Stenosis/Atresia }\end{array}$ & $\begin{array}{l}\text { Attachment to } \\
\text { Atretic Plate }\end{array}$ & $\begin{array}{l}\text { Jahrsdoerfer } \\
\text { Score }\end{array}$ & $\begin{array}{l}\text { Intraoperative } \\
\text { Reconstruction }\end{array}$ \\
\hline 1 & 8 & $M$ & $\mathrm{R}$ & $\mathrm{Y}$ & $\mathrm{Y}$ & $\mathrm{Y}$ & $N$ & $\mathrm{Y}$ & 7 & Y-PORP \\
\hline 2 & 9 & M & L & Y & Y & Y & $\mathrm{N}$ & Y & 8 & Y-No PORPa \\
\hline 3 & 9 & $\mathrm{~F}$ & $\mathrm{R}$ & Y & Y & Y & $\mathrm{N}$ & Y & 7.5 & Y-PORP \\
\hline 4 & 4 & M & $\mathrm{R}$ & $Y$ & Y & Y & $\mathrm{N}$ & Y & 7 & Y-PORP \\
\hline 5 & 3 & M & $\mathrm{R}$ & Y & Y & Y & $\mathrm{N}$ & $Y$ & 6 & Y-PORP \\
\hline 6 & 6 & $M$ & L & Y & Y & Y & $\mathrm{N}$ & $\mathrm{N}$ & 6 & Y-PORP \\
\hline 7 & 11 & M & $\mathrm{R}$ & Y & Y & Y & $\mathrm{N}$ & $\mathrm{N}$ & 7.5 & Y-PORP \\
\hline 8 & 6 & M & $\mathrm{R}$ & Y & Y & Y & $\mathrm{N}$ & $Y$ & 7.5 & Y-PORP \\
\hline 9 & 23 & M & L & $\mathrm{Y}$ & Y & Y & $\mathrm{N}$ & Y & 6 & Y-PORP \\
\hline 10 & 5 & M & $\mathrm{R}$ & $Y$ & $Y$ & $Y$ & $\mathrm{~N}$ & $\mathrm{~N}$ & 7 & No surgery \\
\hline 11 & 8 & $\mathrm{~F}$ & R & Y & Y & Y & $\mathrm{N}$ & $Y$ & 6.5 & No surgery \\
\hline 12 & 11 & $\mathrm{~F}$ & $R$ & $Y$ & $Y$ & $Y$ & $\mathrm{~N}$ & $Y$ & 6.5 & No surgery \\
\hline
\end{tabular}

Note:- Pt indicates patient; L, left; R, right; $Y$, yes; N, no.

${ }^{a}$ No PORP was placed because the incudostapedial joint was intact.
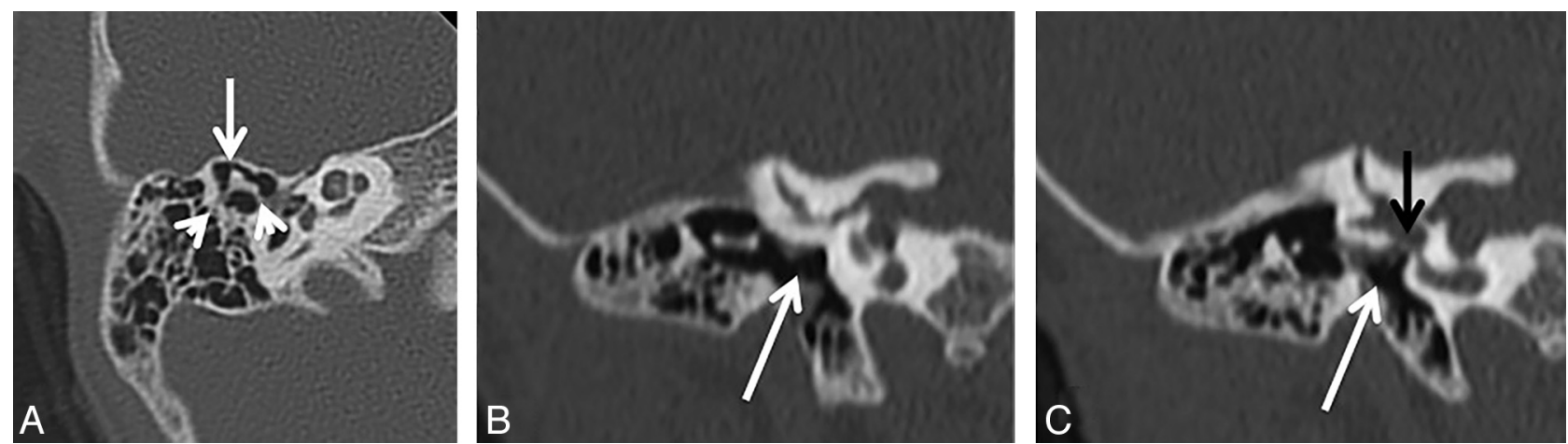

FIG 2. Axial $(A)$ and coronal HRCT ( $B$ and $C$ ) images in patient 5 demonstrate the boomerang deformity (white arrow in $A$ ) and the attachment of the medial and lateral ends of this boomerang complex to the tympanic segment of the facial nerve canal medially and the atretic plate laterally (white arrowheads in $A$ ). Two sequential coronal images ( $B$ and $C$ ) show the absence of the incudostapedial articulation in its expected location (white arrow in $B$ and $C$ ) adjacent to the oval window. The oval window appears patent (black arrow in $C$ ). The findings were confirmed at surgery. Additional findings of an absent stapes capitulum with the presence of the rest of the stapes suprastructure were also noted at surgery.
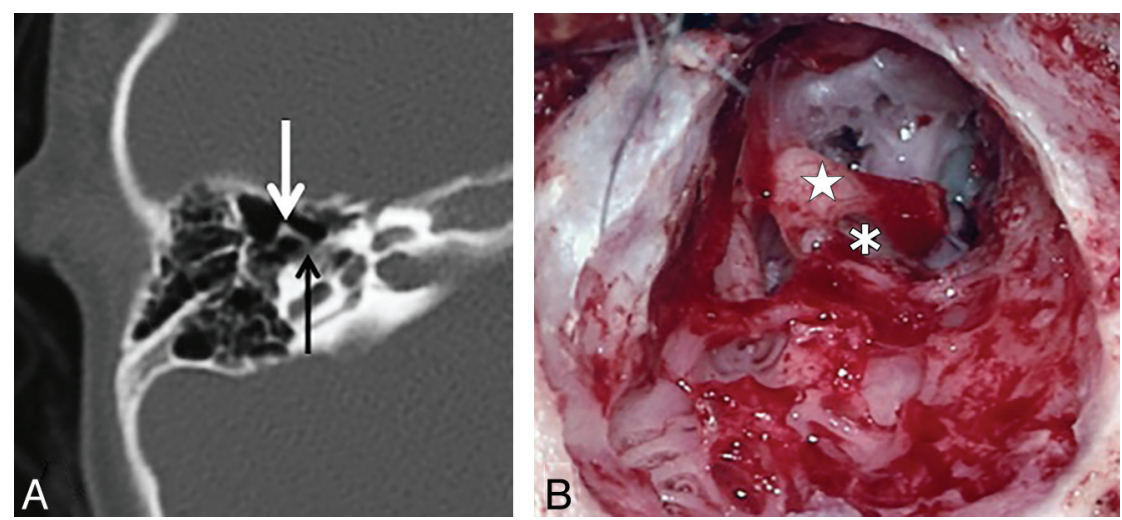

At surgery, the dysmorphic malleusincus complex was as described-a dominant incus with its body fused to a hypoplastic malleus head with absence of the neck, manubrium, and umbo (Figs 3 and 4). The long arm of the incus was attached to the bony tympanic facial nerve canal by a fibrous band; and the absent stapes suprastructure was confirmed in all 9 patients (Fig 3). The anterior position of the facial nerve at the second genu and early mastoid segment was confirmed at surgery in all patients, and the genu

FIG 3. Axial HRCT $(A)$ and intraoperative $(B)$ images in patient 8 showing the fused malleus-incus complex in the right ear. Note the characteristic shape on both axial HRCT (arrow in $A$ ) and intraoperative (white star in $B$ ) images. This intraoperative image also shows a fibrous band extending medially from the complex toward the tympanic segment of the facial nerve canal (black arrow in $A$, white asterisk in $B$ ).

window. Moreover, the stapes capitulum was radiographically absent in all cases, and this finding was confirmed in 9 patients undergoing surgery. The remainder of the stapes suprastructure was intact. The oval window was patent in all patients (Fig 2), and the facial nerve took a somewhat anterior course in the second genu and mastoid segments. No inner ear abnormalities were identified. seemed to take a more acute angle than normal, but this was not measured. The ossicular deformity required removal of the boomerang malleus-incus complex with partial ossicular replacement prosthesis reconstruction in 8 of 9 patients. The PORP was not placed in 1 patient due to a fibrous attachment between the incus and stapes that was thought to be sufficient for sound transmission. No surgical complications, including sensorineural hearing loss, facial nerve injury, or labyrinthine injury, were noted.

With a mean follow-up of 28 months after surgery, patients 

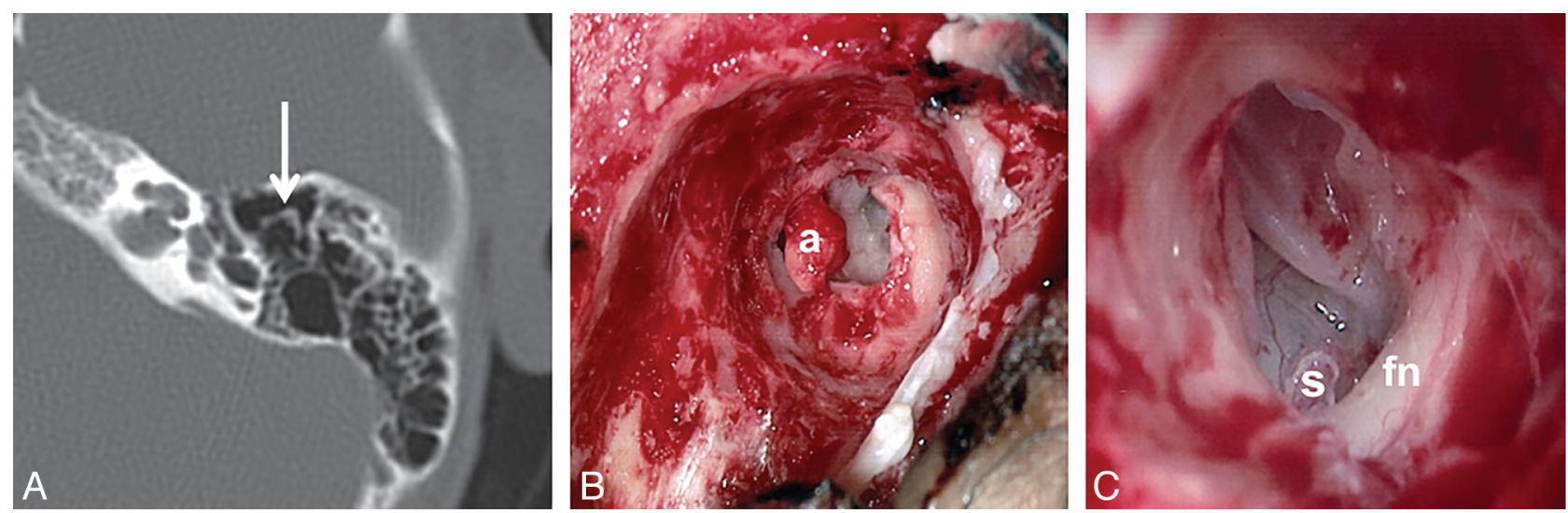

FIG 4. Axial HRCT images $(A)$ in patient 9 show the boomerang complex in the left atretic ear. Intraoperative images $(B$ and $C)$ demonstrate the boomerang complex (a) and a dysmorphic stapes capitulum (S) after removal of the boomerang complex in $C$. fn represents the tympanic segment of the facial nerve.

Table 2: Audiometric data for patients with a boomerang malleus-incus

\begin{tabular}{|c|c|c|c|c|c|c|c|}
\hline & Preop PTA & Preop SRT & Preop ABG & Postop PTA & Postop SRT & Postop ABG & Follow-Up (mo) \\
\hline \multicolumn{8}{|c|}{ Patient No. } \\
\hline 1 & 72 & 65 & 69 & 32 & 25 & 30 & 24 \\
\hline 2 & 57 & 50 & 55 & 32 & 25 & 22 & 58 \\
\hline 3 & 72 & 65 & 59 & 33 & 35 & 25 & 36 \\
\hline 4 & 57 & 50 & 42 & 18 & 10 & 16 & 43 \\
\hline 5 & 65 & 60 & 60 & 42 & 40 & 37 & 33 \\
\hline 6 & 77 & 70 & 74 & 20 & 15 & 7 & 4 \\
\hline 7 & 65 & 40 & 50 & 43 & 35 & 23 & 1 \\
\hline 8 & 67 & 65 & 59 & 27 & 25 & 24 & 53 \\
\hline 9 & 73 & 65 & 48 & 28 & 25 & 8 & 1 \\
\hline Average & 67.2 & 58.9 & 57.3 & 30.5 & 26.1 & 21.3 & 28.1 \\
\hline
\end{tabular}

Note:-Preop indicates preoperative; Postop, postoperative; PTA, pure tone average; SRT, speech reception threshold; ABG, air-bone gap.

enjoyed significant improvement in hearing after surgery, with pure tone average improving $36.7 \mathrm{~dB}$, speech reception thresholds improving $32.8 \mathrm{~dB}$, and the air-bone gap improving $36 \mathrm{~dB}$ (Table $2)$. Average postoperative air-conduction pure tone average, speech reception thresholds, and air-bone gap were 30.5, 26.1, and 21.3 , respectively.

\section{DISCUSSION}

Surgical repair of congenital aural atresia is a challenging operation, which relies ultimately on choosing appropriate candidates for surgery. Not all patients with congenital aural atresia are candidates for surgery to open the ear canal to improve hearing. $\mathrm{Pa}$ tients must have normal inner function as demonstrated by boneconduction audiometry, and the anatomy of the middle ear and its structures must be favorable to support the operation. HRCT is critical in the anatomic and surgical evaluation of patients with congenital aural atresia. ${ }^{1}$

Most patients, including those in this study, with CAA have normal inner ear function because of the different embryologic derivation of the inner ear (otocyst) from the middle and outer ear (branchial apparatus). Some children with CAA in the setting of a named syndrome do have inner ear abnormalities. ${ }^{3}$ The children in this study were nonsyndromic, with normal inner ear morphology on CT and normal inner ear function as evidenced by normal bone-conduction thresholds on audiometry. CAA may stem from an arrest in the embryologic development of the first and possibly second branchial arches. The most common middle ear configuration seen is a malleus-incus complex fused to the atretic plate/lateral middle ear wall, with a normal incudostapedial joint, stapes bone, and oval window. The facial nerve often takes a more acute turn at the second genu and is more anterior. The nerve can also course more laterally in the shortened mastoid segment.

The anatomic appearance of the middle ear and its structures on HRCT offers the surgeon an opportunity to anticipate the patient's middle ear anatomy and to prognosticate to the patient and family the chances of hearing improvement with surgery. To determine surgical candidacy, Jahrsdoerfer developed a 10-point grading scale more than 2 decades ago by using the CT imaging appearance of the middle ear structures and the outer ear. ${ }^{4} \mathrm{~A}$ point is awarded on the basis of the appearance of each anatomic structure, with 2 points awarded for the stapes bone. The Jahrsdoerfer grading scale does have prognostic significance for hearing outcomes-scores $7 / 10$ or higher have a very favorable outcome with normal or near-normal hearing in $85 \%-90 \%$ of these patients. ${ }^{5}$ In the current patient population, 6 of 9 patients scored $\geq 7$ on the scale, indicating a favorable outcome.

Here we describe an unusual configuration of the malleusincus complex, in which the malleus and incus, fused in all patients with aural atresia, have a boomerang appearance and the incus has a fibrous attachment to the fallopian canal and is not attached to the stapes bone. In addition, the stapes capitulum is absent and the suprastructure is small. The boomerang corresponds anatomically to the short process, body, and long process of the incus, the dominant ossicle, with no cleavage plane or joint 
space between the body of the incus and the very hypoplastic malleus head. There is no malleus neck, manubrium, or umbo. These findings were corroborated at the time of surgery.

The boomerang configuration most likely represents a more severely hypoplastic malleus bone, possibly from an arrest earlier in the embryologic development of the first branchial arch than is normally seen in patients with aural atresia. This characteristic finding certainly has implications for surgical repair.

When this anatomic anomaly is recognized, the surgeon can anticipate possibly needing to use a partial ossicular replacement prosthesis placed over a rudimentary stapes bone. Hearing results may not be as good as those of conventional atresia surgery compared with when the patient's native ossicular chain is used. ${ }^{2}$ However, hearing results in the current study are comparable with those of other reports, with the postoperative pure tone average in the mild hearing loss range and speech reception thresholds in the borderline normal range. .,4-8 $^{2}$

In addition, the PORP reconstruction in which the prosthesis is placed on the mobile stapes crural arch appears to be more stable and reliable than the PORP reconstruction in which the prosthesis is placed through the obturator foramen down onto the stapes footplate. ${ }^{5}$ Other studies have reported comparable results between intact native ossicular chain reconstruction and ossicular replacement prostheses. ${ }^{6}$ The method of reconstruction is dictated by the anatomy at surgery, but if the stapes suprastructure is intact and mobile, the author has found superior results from placing the prosthesis on the suprastructure. ${ }^{2}$

One limitation of this study is the lack of long-term data (mean follow-up hearing data are 28 months after surgery; range, 1-58 months). Patients come to the University of Virginia for surgery from around the country. Obtaining long-term hearing outcome data has been a challenge in this population.

This data is important when counseling patients and their families on the possibility of successful hearing gain with canaloplasty surgery. While early short-term hearing results appear favorable with this reconstruction in this anomaly, long-term stability of hearing remains to be demonstrated.

\section{CONCLUSIONS}

Recognizing the boomerang sign in the middle ear in patients with congenital aural atresia carries implications for the otologic surgeon and for potential hearing outcomes after surgery. This anomaly is almost always associated with other abnormalities of the middle ear, including an absent or dysmorphic stapes capitulum and ossicular discontinuity, with a fibrous attachment between the long process of the incus and the fallopian canal. The boomerang malleus-incus is commonly associated with an abnormal capitulum, but due to the presence and mobility of the remainder of the stapes, patients with this anomaly may undergo successful PORP placement. Preoperative identification by the radiologist with communication to the referring otologist helps in optimal clinical decision-making, surgical planning, and patient counseling.

Disclosures: Bradley W. Kesser—RELATED: Patents: Royalties: less than $\$ 200$ per year for an ear simulator that he and his colleagues patented and licensed to Nasco Inc for educational purposes.

\section{REFERENCES}

1. Gassner EM, Mallouhi A, Jaschke WR. Preoperative evaluation of external auditory canal atresia on high-resolution CT. AJR Am J Roentgenol 2004;182:1305-12

2. Dobratz EJ, Rastogi A, Jahrsdoerfer RA, et al. To POP or not: ossiculoplasty in congenital aural atresia surgery. Laryngoscope 2008;118: 1452-57

3. Vrabec JT, Lin JW. Inner ear anomalies in congenital aural atresia. Otol Neurotol 2010;31:1421-26

4. Jahrsdoerfer RA, Yeakley JW, Aguilar EA, et al. Grading system for the selection of patients with congenital aural atresia. Am J Otol 1992;13:6-12

5. Shonka DC, Jahrsdoerfer RA, Kesser BW. The Jahrsdoerfer grading scale in surgery for congenital aural atresia. Arch Otolaryngol Head Neck Surg 2008;134:873-77

6. Chang H, Song JJ, Choi BY, et al. Partial ossicular replacement versus type II tympanoplasty in congenital aural atresia surgery: a matched group study. Otol Neurotol 2009;30:609-13

7. Lambert PR. Congenital aural atresia: stability of surgical results. Laryngoscope 1998;108:1801-05

8. De la Cruz A, Teufert KB. Congenital aural atresia surgery: long term results. Otolaryngol Head Neck Surg 2003;129:121-27 